\title{
METHODOLOGY FOR POROSIMETRY IN VIRTUAL CEMENTITIOUS COMPOSITES TO ECONOMICALLY AND RELIABLY ESTIMATE PERMEABILITY
}

\author{
KAI Li ${ }^{\bowtie}$, PIET StROEVEN AND NGHI LB LE \\ Faculty of Civil Engineering and Geosciences, Delft University of Technology, Stevinweg 1, 2628CN Delft, \\ the Netherlands \\ e-mail: K.Li-1@tudelft.nl; P.Stroeven@tudelft.nl; L.B.N.Le@tudelft.nl \\ (Received January 12, 2015; revised May 21, 2015; accepted May 25, 2015)
}

\begin{abstract}
A novel methodology is described for porosimetry as well as for water transport through the pore system in dynamic DEM-based virtual cementitious materials. The pore network topology, the pore size distribution and the pore connectivity are assessed on the basis of a robotics-inspired pore delineation method and star volume measurements. Permeability estimates are based on a tube network model that incorporates these parameters and a shape factor. Since concrete contains in practical situations a variable amount of water, permeability estimation is presented as a function of the state of saturation. Satisfactory agreement is found with experimental data, validating the methodology. Earlier, the various "building blocks" were separately validated.
\end{abstract}

Keywords: DEM, permeability, porosimetry, water saturation.

\section{INTRODUCTION}

Transport-based durability problems still constitute complicated issues in experimental researches. Water permeability testing of the real concrete ("realcrete") is quite laborious, time-consuming and thus expensive. Speeding up the transport process introduces uncertainties as to the interpretation of obtained results. Therefore, the most common experimental approach in concrete technology is by MIP (Mercury Intrusion Porosimetry) (e.g., Abell et al., 1999). The instrumentation is relatively cheap and as a consequence available in many research laboratories in the field of concrete technology. The experiments can be directly interpreted in terms of the three-dimensional (3D) pore size distribution (PoSD). Unfortunately, it has been demonstrated that a wide gap can exist between image analysis and MIP data, as Fig. 1 may prove (Diamond, 2000). Very narrow pathways in the pore system (so called "bottle necks") prevent passage of the pressurized mercury making part of the pore network inaccessible. The surface layer of the concrete specimens can also lead to limited inaccessible of the mercury to the underlying part of the pore network structure. This are two widely accepted deficiencies of the approach giving rise to the biased PoSD information as compared to the image analysis results.
An incidentally used alternative is WMIP, (Wood's Metal Intrusion Porosimetry) (Willis et al., 1998). Based on the same principles, it additionally renders possible stabilizing the specimen through solidification of the used intrusion material. Hence, sections can thereupon be subjected to quantitative image analysis.

Quantitative image analysis is incidentally pursued for porosimetry (Scrivener, 1989; Lange et al., 1994; Wang and Diamond, 1995; Hu and Stroeven, 2003), however the pore gradient structures resulting from the formation of the relatively porous Interfacial Transition Zones (ITZs) near the surfaces of the aggregate grains complicate the sampling operation; it thereby augments the time-consuming character of the analysis. In $\mathrm{Hu}$ (2004) and Stroeven et al. (2010) methods for 3D interpretation of $2 \mathrm{D}$ section images are reviewed, so the interested reader is referred to these publications focusing on experi-mental strategies to porosimetry.

Today it is readily possible economically producing realistic representations of the real-create by means of advanced computer simulation methods. The pore network system developed after hydration simulation in the virtual computer-made concrete ("compucrete") is thereupon available for quantitative geometrical and topological studies. Information like porosity, connected fraction of porosity, PoSD and pore tortuosity can be 
obtained. This would permit representing the pore network structure by a tube system that incorporates the aforementioned information. Common hydraulic modelling strategies finally yield permeability information. This is demonstrated herein.

Unfortunately, most popular approaches in concrete technology produce compucrete by RSA (Random Sequential Addition) particle packing strategies, such as by $\mu$ ic (Bishnoi and Scrivener, 2009) or by Hymostruc3D (Breugel, 1991). A modern porosimetry method developed by Ye (2003) for 3D reconstruction from serial sections of pore space in the hardened virtual cement paste is based on Hymostruc3D. Although it is basically possible directly deriving 3D information on PoSD from the virtual cementitious specimen, the results suffer from the deficiencies of RSA. This and other simulation methods including stochastic modelling approaches are discussed in Stroeven et al. (2010).

Two building blocks of a new modern methodology for porosimetry on virtual cementitious pastes incorporating many stereological issues - were earlier published in IA\&S (Stroeven et al., 2012a,b). Since the pursued engineering information is basically of structure-sensitive nature, the packing of particles (aggregate and cement grains alike) should be conducted by DEM (Discrete Element Method) and not by either one of the in concrete technology popular RSA systems, since they produce virtual material with biased dispersion. As a consequence, fracture (He et al., 2011) or durability estimates (see this publication) would be inevitably biased, too.

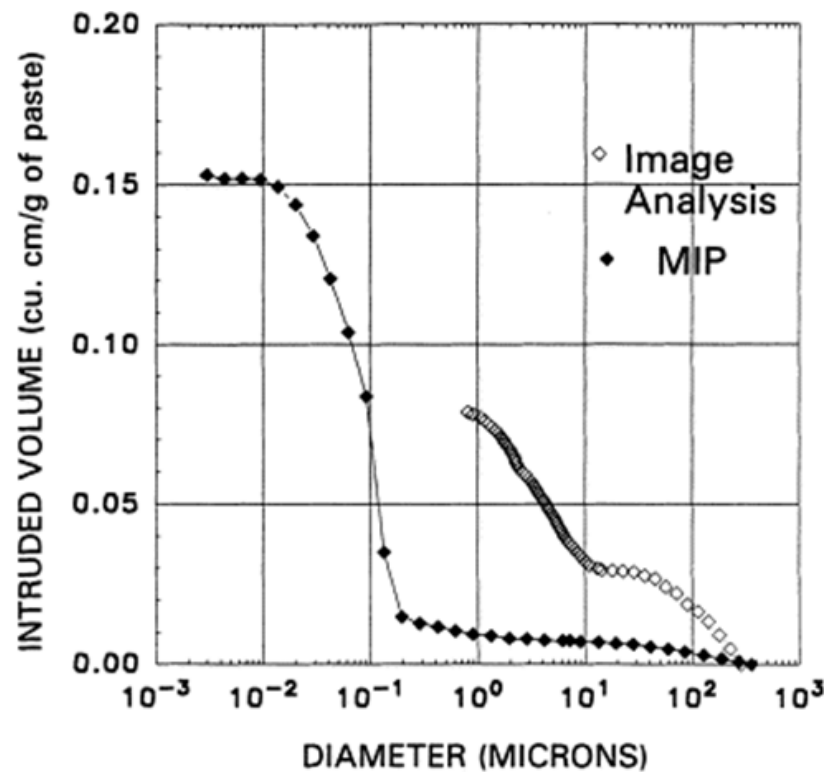

Fig. 1. Differences between porosimetry results obtained by image analysis and by MIP obtained on similar cementitious material samples (Diamond, 2000).
The dynamic force-based DEM system used in the presented research (Habanera's Discrete Element System, HADES) has already been described elsewhere (He, 2010; Stroeven et al., 2011), so for details the reader is referred to these publications. The new vector-based (i.e., geometric primitivesbased) hydration simulation system, XIPKM, has also been published earlier (Le et al., 2013). It is an extension of the well-known IPKM method commonly employed in producing analogue versions of the hardened material. See, e.g., Navi and Pignat (2005). In alternative approaches, a digitized version of the material is produced and porosimetry is adapted to such a digitized set up. For representative studies, one is referred to work by NIST collaborators (e.g., Garboczi and Bentz, 2001). Analogue approaches have however major advantages. Particularly, the scale sensitivity of the digitized approach constitutes a serious drawback. Moreover, uniform randomness (UR) in quite densely packed cement particle systems will not constitute a reliable representation of the realcrete. Hence, it was demonstrated earlier by Chen et al. (2006) that for similar cases, the pore depercolation processes during hardening were distinctly different for the DEM, RSA and digitized approaches.

So, the proposed strategy represents an analogue methodology for production of the material and of a quantitative assessment of pore network characteristics. It is readily available for application to engineering problems whereby durability constitutes a highly relevant issue. So far, all simulation systems assume cement grains to be spherical, the present one included. Recently, it was demonstrated however that real cement grains are more polyhedron-shaped (Garboczi and Bullard, 2004). This would complicate simulating particle interferences on micro-level in a hydrating system, however. Therefore, we are developing an algorithm for nano-level particle packing of the outer layer of calcium silicate hydrate (CSH) (Li et al., 2014). This would render possible assuming cement particles to be poly-hedron-shaped. Moreover, surface roughness would be implemented on pore surfaces reducing conductance for water transport. This will be touched upon in the discussion section.

Of course, the permeability outcomes will be validated on the basis of available experimental data. Reliability of the various building blocks is validated in the various publications referred to. The internal moisture conditions of concrete thereby play a major role. This gives rise to partly saturated conditions in the pores that can lead to permeability data varying over three to four orders of magnitude. The controlling of this state is also a factor seriously hampering 
the reliability of the outcomes of physical experiments that are potentially available for validation purposes.

\section{POROSIMETRY IN VIRTUAL CONCRETE}

In general, five building blocks or stages of the proposed methodology can be distinguished. The first four are developed for porosimetry and will be briefly reviewed in what follows, since they are explicitly described in the international literature to which will be referred. Such publications also review other methods for porosimetry, i.e., experimental studies, stochastic approaches and computer simulation methods, both DEM- as well as RSA-based. The successive building blocks are: particle packing by DEM, hydration simulation by XIPKM, pore delineation by DRaMuTS and pore measuring by SVM. The remaining block for permeability estimation will be discussed in the appropriate section.

\section{DEM-BASED PARTICLE PACKING}

A dynamic force-based DEM denoted HADES is developed for packing simulation of basically artificially-shaped particles on meso-, micro- and nanolevel. This is the first building block for porosimetry, but it was used as well on meso level for packing of non-spherical particles representing crushed rock aggregate (He, 2010). Application on micro-level, so far, involved spherical particles, because of the complications arising from interferences of hydrating non-spherical particles. We are presently exploring simulation of the outer CSH hydration layer on nanolevel. This would, on the one hand, eliminate coping with interference problems by the vector (i.e., geometry-based) approach. On the other hand, more realistic, polyhedron-types of particles could be considered (Garboczi and Bullard, 2004).

In the present case, the sequence of activities encompassed the following. As commonly done, a Rosin-Rammler function is specified to represent the particle size distribution of the Portland cement; i.e., $G(d)=1-\exp \left(-\mathrm{b} d^{\mathrm{a}}\right)$, whereby $G(d)$ stands for the mass or volume fraction of the cement passing a sieve with opening $d$, and $\mathrm{a}$ and $\mathrm{b}$ are constants $(\mathrm{Hu}, 2004)$. Mineral admixture particles will have their own grain size distribution.

The amount of cement and of the mineral admixture necessary to achieve the required water to binder ratio and blending percentage are assessed and the associated particles are dispersed by RSA procedure in an enlarged container, generally with two rigid and four periodic boundaries (Fig. 2). The two opposite rigid boundaries are contrasted by green colour in this figure. This set up is selected to simulate the packing of the binder between neighbouring aggregate grain surfaces. Otherwise, six periodic boundaries are selected for simulating bulk material. Next, particles are set to linearly move and rotate according to Newtonian rules. When the afore-mentioned global state of particle density is reached, the dynamic stage is terminated. Note that global density can be as high as 0.6 (so close to random loose packing) for very low water to binder ratios applied in the (super) high performance range of concrete qualities.

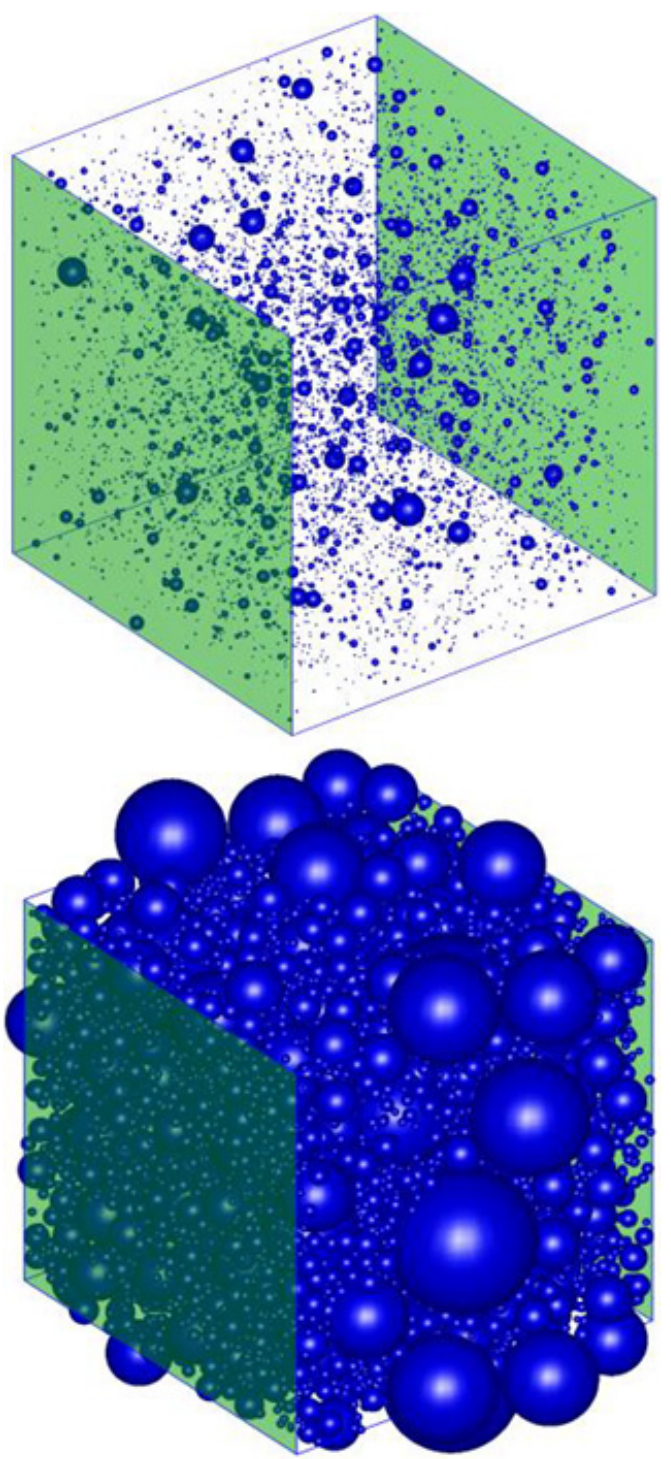

Fig. 2. Dilute dispersed particles in enlarged container at the top (initial stage) are densely packed at end of the dynamic stage (bottom picture). Green walls represent the rigid boundaries, while the rest are of the periodic type. For visualization purposes, the compacted con-tainer is shown on appropriately enlarged scale 


\section{HYDRATION SIMULATION BY XIPKM}

XIPKM stands for eXtended Integrated Particle Kinetics Method for the simulation of a fresh multicomponent blended cement system (Le et al., 2013). It is the second building block of the porosimetry methodology. The IPKM reference method that only considers the $\mathrm{C}_{3} \mathrm{~S}$ (tricalcium silicate) compound in the Portland cement (PC) is described in Navi and Pignat (2005). For details of the method the reader is therefore referred to this paper. The new method adds to the $\mathrm{C}_{3} \mathrm{~S}$ the missing three compounds of the $\mathrm{PC}$, i.e., dicalcium silicate, tricalcium aluminate and tetracalcium aluminoferrite. In this paper, they are denoted as $\mathrm{C}_{2} \mathrm{~S}, \mathrm{C}_{3} \mathrm{~A}$ and $\mathrm{C}_{4} \mathrm{AF}$, respectively, in accordance with the Cement Chemist Notation $(\mathrm{CCN})$.

Basically, XIPKM takes the four major cement compounds of the PC in consideration, as shown schematically in Fig. 3. They are modelled as spherical sectors in proportion to the chemical composition of the cement. These sectors react differently with water during the hydration process. The hydration rate is established separately for the reaction front of each phase $\lambda$ of the multiphase particle. The hydration product $\mathrm{CSH}$ covers the sectors until the original size of the cement grain is recovered. This inner layer $\left(\mathrm{CSH}_{\mathrm{in}}\right)$ is denser than the outer layer (denoted $\mathrm{CSH}_{\text {out }}$ ) formed on top of it during continuing hydration. This concept is in agreement with experimental observations about structure formation in hydrating cement-based pastes (Richardson, 2004). Of course, the consumption of water by the different compounds during the hydration process is followed in detail and compared with the available amount of water.

The spherical fronts of the particles that expand as a result of continuing hydration lead to complicated interference problems. This is solved by a voxelbased approach, which offers a better solution than by the vector approach, as schematically indicated in Fig. 4. The produced $\mathrm{CH}$ (calcium hydroxide) volume during hydration of the cement particles causes nucleation of new $\mathrm{CH}$ particles and precipitation on the surfaces of the existing $\mathrm{CH}$ particles. The latter as well as the CAH (hydrogarnet) and FH (iron hydroxide) are assumed forming randomly dispersed spheres in the pore solution.

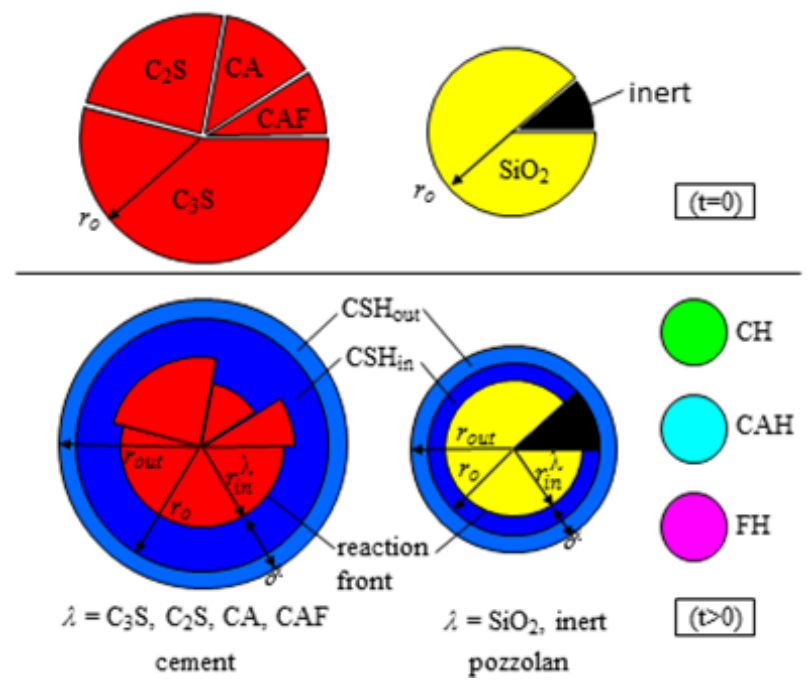

Fig. 3. Particle models of cement, pozzolanic admixture and hydration products in XIPKM (Le, 2015).

In case the paste is blended with a pozzolanic admixture, part of the produced $\mathrm{CH}$ volume will serve as a reactant in the pozzolanic hydration. The mineral admixture is supposedly composed of silica and an inert portion (example is rice husk ash). The silica reacts with (part of) the lime in the water. A specimen of hardened blended cement is displayed in Fig. 5. This vector-based approach yields an analogue representation of the virtual material.

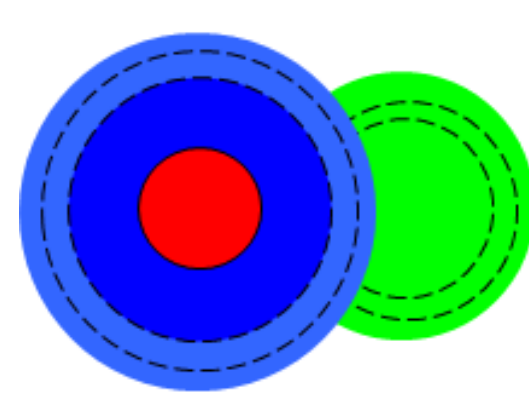

(a) vector approach

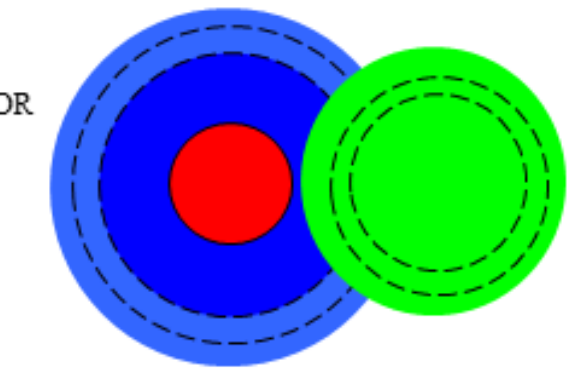

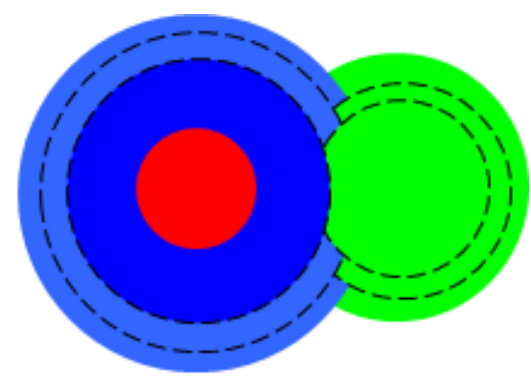

(b) voxel approach

Fig. 4. Phase representation at layer-by-layer overlaps of two neighbouring particles (of which one is presented in detail, showing its unhydrated nucleus, and CSHin and CSHout layers) during the expansion due to hydration of the particles. 


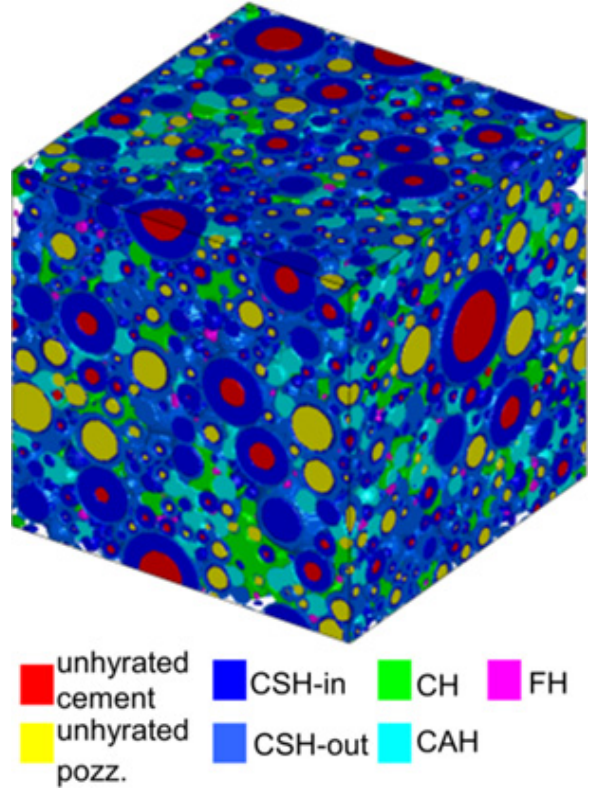

Fig. 5. 3D visualization of microstructure and corresponding pore space of a blended cement paste by a digital image based technique or voxel system. Each unhydrated multiphase core is represented by a sphere whose volume equals the total volume of the phases.

\section{PORE DELINEATION BY DRAMUTS}

DRaMuTS, the third building block of the porosimetry methodology, stands for a Double Random Multiple Tree Structuring system for delineating the complete pore network and for topology assessment (Stroeven et al., 2012a). By this method, randomized data structures are built incrementally in two stages. The first stage is to rapidly explore the pore space. Virtual trees consisting of nodes and lines connecting pairs of such nodes (like branches in real trees), grow randomly and incrementally in the pore medium. To speed up the process, deviating from the rapidly-exploring random tree approach, developed in robotics, a point is shifted to avoid rejections, thereby however violating the uniform randomness of the tree system. When neighbouring trees are developing in the same pore, they are forced to merge. In the second stage, a system of probing points is generated uniformly at random in pore space. As a result, such points can be used for statistical assessment of pore characteristics in which point classifications are realized by connections of such points to the tree systems. For example, the connected fraction of pores can be estimated by the fraction of the total number of points that can be associated with the percolated tree branches. Fig. 6 presents the pore network structure as obtained by DRaMuTS. At the top all pore trees are displayed, at the bottom only those connecting outer surfaces of the simulated specimen (the so called main trunks).
Note that different colours in Fig. 6 (top) represent the various trees growing from different seeds. These seeds can be generated on both top and bottom surfaces of the cube to speed up the calculation. The pores that are delineated by zigzag lines are smoothed by mathematical operations so that they can be used for permeability estimation by a network model.
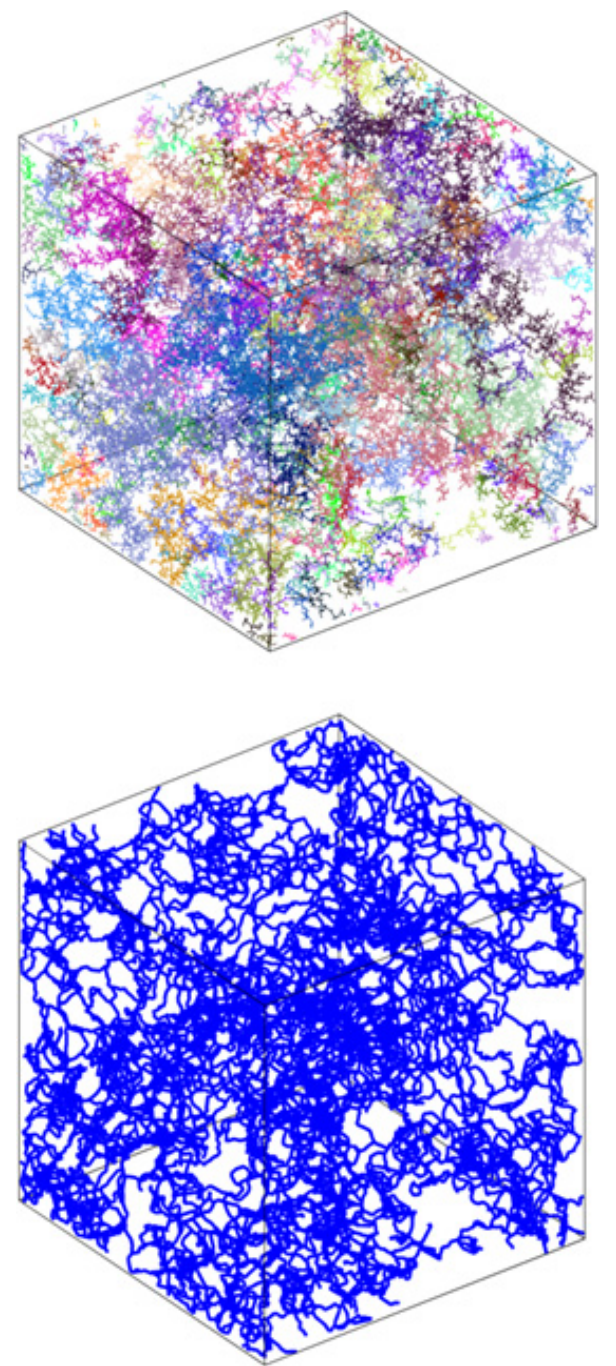

Fig. 6. Pore delineation in $100 \mu m$ cubes for a plain $P C$ sample. The various trees are characterized by different colors. All trees growing in capillary pores are shown (top) as well as the extracted main trunks only (bottom).

\section{PORE GEOMETRY AND TOPOLOGY}

Topology of the pore network structure is already assessed on the basis of the tree morphology. Thereupon, star volume measuring (SVM) in UR dispersed points inside the pore network is applied (Stroeven et al., 2010). This is the fourth building block for porosimetry. The PoSD is obtained from SVM whereby the UR points are employed as the nuclei of "stars". In each point a system of pikes is generated 
isotropic uniformly at random (IUR) or in systematic directions. Length of a pike, $l_{i}$, spanning in an unobstructed way the distance from the star's nucleus to the nearest pore surface, is assessed. In 3D space, $d_{\mathrm{i}}=2 \sqrt[3]{l_{\mathrm{i}}^{3}}$, whereby $d_{\mathrm{i}}$ is an unbiased estimate of local pore size, i.e, of the size of the representative sphere. Such star volume measurements in the nuclei provide the information for the construction of the 3D volume-based pore size distribution. The number-based PoSD could be derived as well, of course.

Alternatively, a plane is located through a nucleus and pikes are, e.g., designed in uniform directions in the plane section. The area of this pore section is estimated by $A_{\mathrm{i}}=\pi \overline{l_{1}^{2}}$, with $l_{\mathrm{i}}$ as the unobstructed distance from the nucleus to the nearest pore perimeter. By rotating the plane section, the minimum value of $A_{\mathrm{i}}$ can be determined. This is denoted the pore throat area, which represents the area of the representative circle. An adjustment of the sensitivity to a relevant engineering level involves eliminating all throat areas or pore sizes smaller than assumed minimum values (Le and Stroeven, 2012).

Fig. 7 presents for illustrative purposes some porosimetry results of an application to fine-grained rice husk ash (RHA)-blended Portland cement paste. Fig. $7 \mathrm{a}$ is the gradient structure of local porosity in the binder paste between two neighboring aggregate grains. Fig. $7 \mathrm{~b}$ is the volume-based pore size distribution. The effects of partial cement replacement with (in this case 20\%) RHA are obvious: a reduced extension of the more porous ITZ (interfacial transition zone around aggregate grains) and the disproportionate reduction of pore size in the ITZ due to

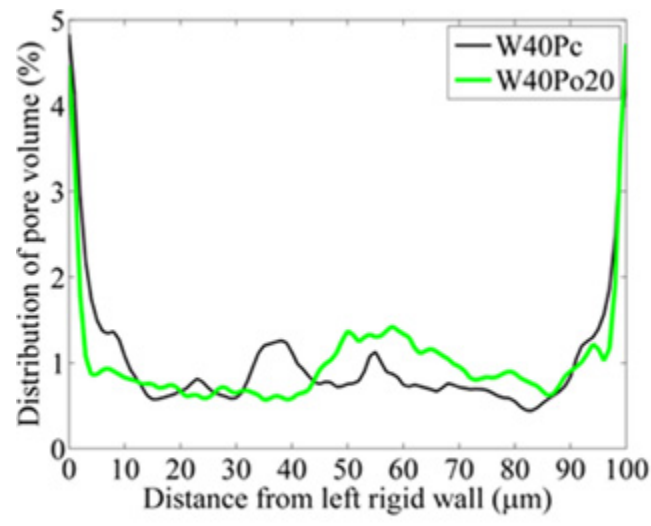

(a) blending. For further details on such research efforts, see (Stroeven and Le, 2013). Note that the observed dimension of the ITZ in the virtual representation depends on the design of the virtual material. This always involves a restriction in the particle range, to limit the computation efforts. Hence, engineering estimates are not straightforwardly obtained.

\section{PERMEABILITY OF VIRTUAL CONCRETE}

\section{DESIGN OF TUBE NETWORK MODEL}

Finally, a network structure of cylindrical tubes and nodes is constructed as an additional building block for permeability estimation. This network structure represent the smoothed main trunks, using all relevant geometric and topological pore network characteristics that are determined. This network structure is supposedly subjected to a given pressure gradient between inlet and outlet nodes. Applying the volume conservation law at nodes between two or more tubes, a set of algebraic equations is obtained with nodal pressures as unknowns. After solving this set of equations, the permeability is estimated by Darcy's law (Le and Stroeven, 2014; Le, 2015) describing the laminar flow of a fluid through a porous medium:

$$
K=\frac{\mu\langle u\rangle L}{\Delta P}
$$

where $K=$ permeability $\left[\mathrm{m}^{2}\right],\langle u\rangle=$ average velocity of the fluid $[\mathrm{m} / \mathrm{s}], L=$ sample length $[\mathrm{m}], \mu=$ dynamic viscosity of the intruding fluid $\left[\mathrm{N} . \mathrm{s} / \mathrm{m}^{2}\right]$, and $\Delta P=$ applied pressure gradient $\left[\mathrm{N} / \mathrm{m}^{2}\right]$.

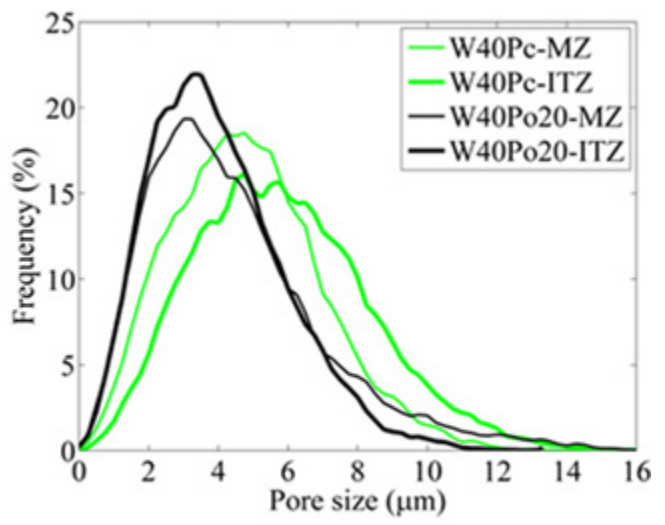

(b)

Fig. 7. (a) Gradient structures between neighbouring aggregate surfaces in local porosity (or pore volume) in plain and blended cement paste obtained by serial sectioning and point counting: porosity in and extent of ITZ are reduced; (b) volume-based pore (=throat) size distribution, moreover, reveals pore size to be disproportionately refined in the ITZ by blending. MZ is middle zone between the ITZs; Pc=Portland Cement; Po20 is $P C$ with 20\% RHA; W40 $=w / b$ or $w / c=0.40$. Results obtained by presented methodology (Le and Stroeven, 2012). 
The flow inside a tube with constant circular cross section is assumed to be slow, saturated, incompressible and laminar according to the Hagen-Poiseuille law. The irregular shapes of the pore cross-sections are still taken into account in estimating the hydraulic conductance of the tubes. Fig. 8 shows the "inefficiency" of a randomly selected cross-sectional shape for transport (Le, 2015).

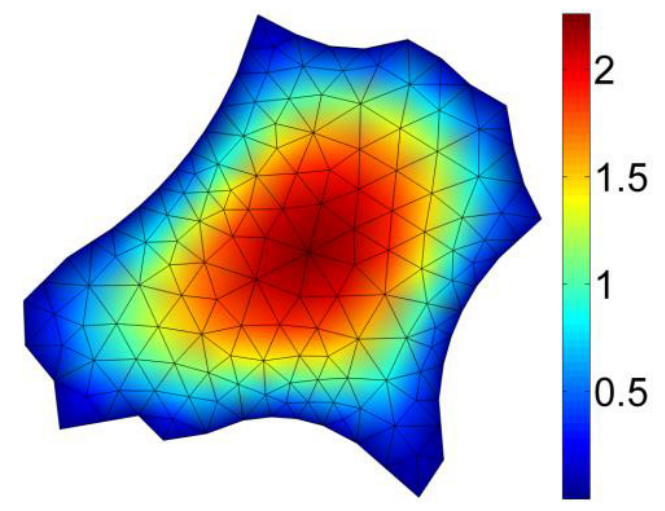

Fig. 8. Relative information on the velocity field of water flow through an irregular cross-section of a pore solved by FEM.

For an ideal circular cross-section, the conductance $\mathrm{C}$ can be derived from the Hagen-Poiseuille's equation and expressed by:

$$
C_{c i r}=\frac{\pi D^{4}}{128 \mu}
$$

where $D$ is the diameter of the circular section. In the section, the flow distribution is parabolic. However, the cross-sections of capillary pores in cementitious porous media are generally as irregularly-shaped as depicted by Fig. 8. The influence of the shape factor should therefore be taken into account for the estimation of cross-sectional conductance. Patzek and Silin (2001) investigated conductance of non-circular cross-sections, i.e., triangular, rectangular and elliptic shapes, in which the cross-sectional conductance is shown to be proportional to the shape factor of the cross-section. The shape factor in such investigations is expressed through the dimensionless 'shape factor' Sh of Mason and Morrow (1991)

$$
S h=\frac{A}{P^{2}},
$$

where $A$ and $P$ denote the cross-sectional area and the perimeter length, respectively. Note that this is inversely proportional to circularity used in concrete technology (Hu and Stroeven, 2006; Stroeven et al., 2009; He, 2010).
The velocity is integrated over the cross-sectional area to obtain the volumetric rate and therefore the hydraulic conductance. Fig. 8 is an example of the velocity field of a cross-section of a pore in a simulated cement paste; the geometrical configuration of the pore section is obtained by the enhanced SVM (Le, 2015). This involves a shift in the location of the random node in the throat section to that of the nucleus of the representative circle. Fig. 9 shows the relation between dimensionless conductance of pore sections (pore throats) in a matured (i.e., hardened) cement specimen and their dimensionless shape factors, in comparison to such relations for random triangles and quadrilaterals. Obviously, all values of conductance as well as shape factor $S h$ are smaller than those of the circular section, i.e., $\tilde{C}_{c i r}=1 / 8 \pi \approx$ 0.04 and $S h_{\text {cir }}=1 / 4 \pi \approx 0.08$.

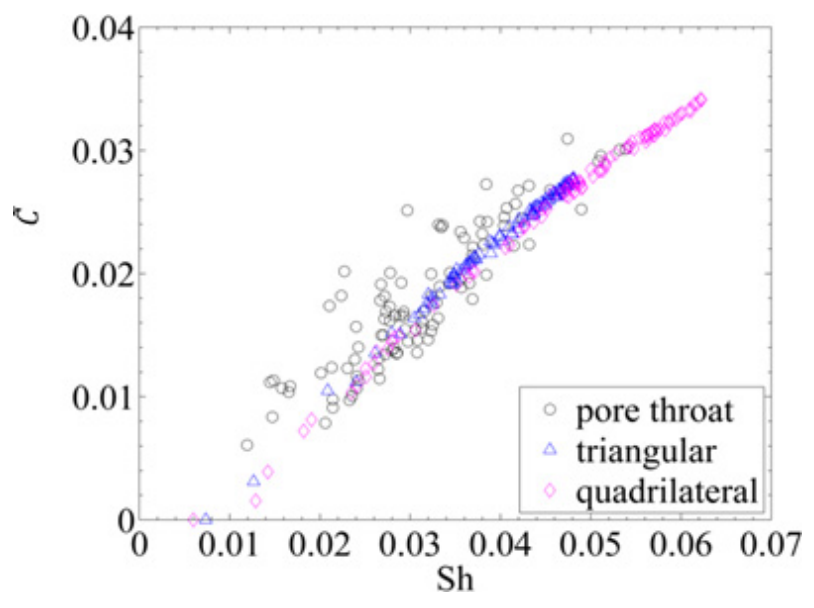

Fig. 9. Dimensionless hydraulic conductance versus shape factor for 100 pore cross-sections (throats) of a simulated cement paste; 100 random triangles and 100 random quadrilaterals.

To obtain the conductance of all equivalent tubes, the cross-sectional hydraulic conductance can be calculated at the pore throats along the main trunks. However, solving the flow by FEM at every pore throat would be not practical. In this study, hence, the influence of the shape factor on conductance is integrated statistically; the dimensionless conductance is estimated by FEM at an adequate number of random points in pore space. The obtained values are then used to form a function yielding the conductance for arbitrary pore throats via its shape factor. For example, the conductance versus shape factor of pore throats is shown (in relative terms with respect to a circular section) and fitted by a linear function as in Fig. 10. Herein, the hydraulic conductance of a pore throat is given by

$$
\widetilde{C}_{p o}=\widetilde{C}_{c i r} \zeta
$$


where $\xi$ is the shape factor that equals the ratio of the conductance of the pore throat and of a representative circle, as shown by Fig. 10.

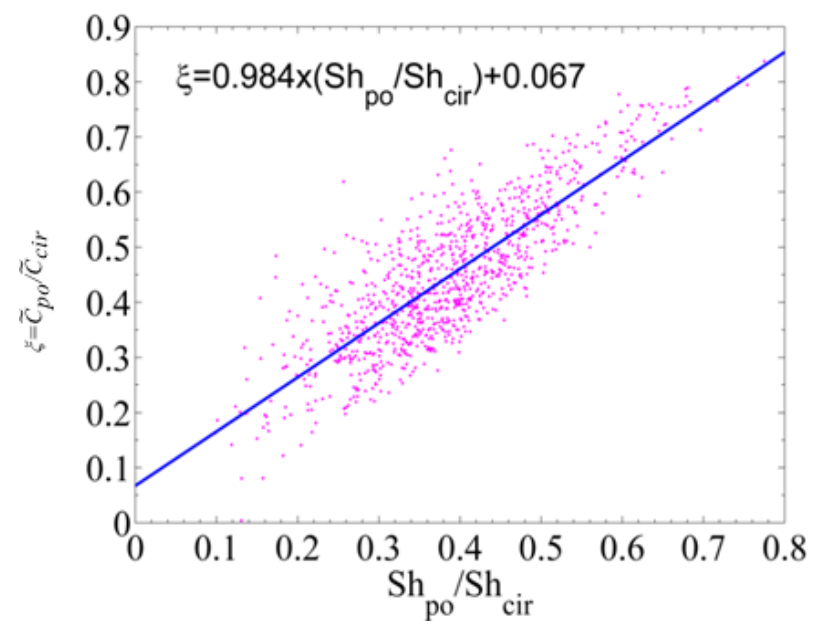

Fig. 10. Function obtained by linear regression. for the conductance of pore throats versus shape factor relative to that of circular cross-sections.

Fig. 10 contains all observations conducted by the presented methodology on Portland cement with different $w / c$ and different fineness. This graph renders possible estimating the pore throat conductance $\widetilde{C}_{p o}$ on the basis of its shape factor $\xi$. The overall conductance of a tube element is determined by averaging local values in a number of randomized points along the tube's length. Of course, the regression line will be influenced by the design of the fresh particle mixture, in particular by the size range involved (Le et al., 2015). Hence, summarizing, we have designed the pore network structure as consisting of nodes and smooth circular tubes. However, the resulting conductance is reduced along the tube lengths in accordance with the actual local shapes of the pore throats.

\section{SATURATED CAPILLARY PORES}

Estimation of permeability $K$ for bulk and ITZ separately demonstrates the well-known relatively large contribution by the latter. The joint $K$ value is however not so much dominated by that of the ITZ, particularly not for moderate or high $w / c$ ratios as can be concluded from Fig. 11. A related discussion can also be found in Stroeven et al. (2012b). This global intrinsic permeability level fluctuates for the given mixtures from $1.453 \mathrm{e}-16 \mathrm{~m}^{2}$ to $3.562 \mathrm{e}-15 \mathrm{~m}^{2}$ and $1.608 \mathrm{e}-15 \mathrm{~m}^{2}$, respectively. This is in agreement with other vector-based simulation outcomes, such as by Pignat et al. (2005), validating the methodology.

Nevertheless, this seems significantly exceeding experimental values. In the first place, however, it should be remarked that mostly use is made of MIP of which Fig. 1 already demonstrated its biased character. Secondly, the set up so far is for fully water saturated pores, under which condition water moves best through the pore network system. Thirdly, even when pursued in experiments, this condition is probably never realized in reality. Hence, it will be shown in the rest of this article that the estimates based on the compucrete "produced" by the presented methodology offers quite reliable estimates for the realcrete.

\section{PARTIALLY WATER-SATURATED PORES}

The modelling platform XIPKM generates the microstructure of the solid phases. The remaining space is usually assumed fully water-filled. This hypothesis is equivalent to the ideal underwater curing; the sample under this condition should be fully saturated, corresponding to a water saturation degree of $100 \%$. However, this ideal situation is difficult to achieve in

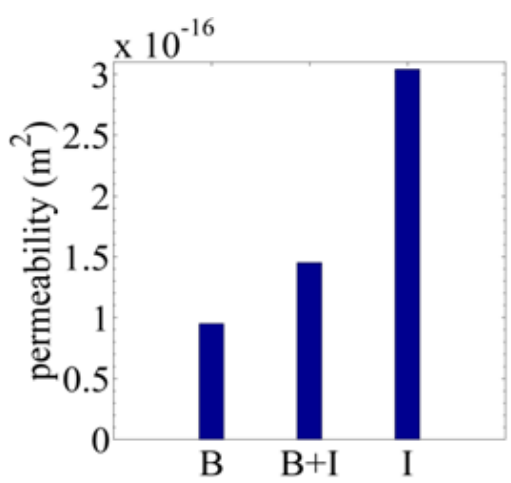

(a) W25F300

( 90 days, $\mathrm{p}=9 \%$ )

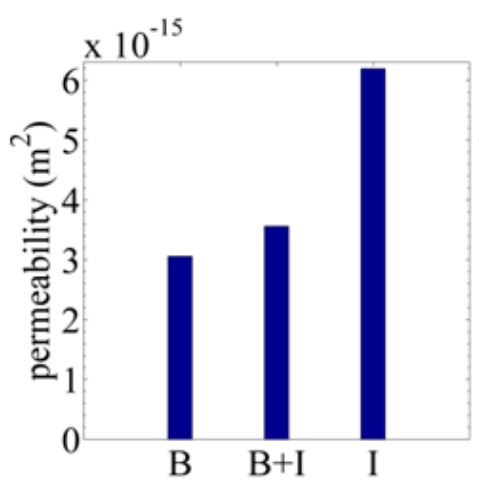

(b) W40F 300

( 90 days, $\mathrm{p}=19 \%$ )

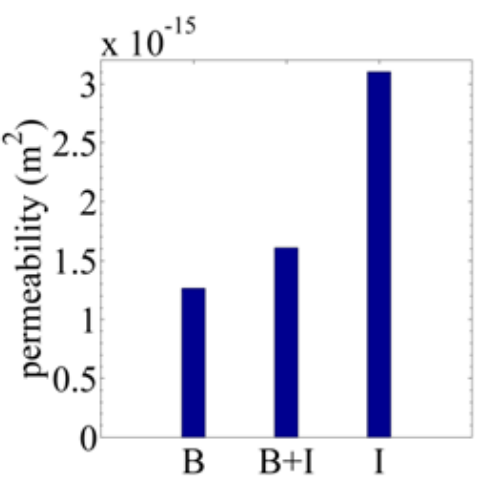

(c) $\mathrm{W} 40 \mathrm{~F} 420$

( 60 days, $\mathrm{p}=19 \%)$

Fig. 11. Permeability of different zones in fully saturated cement paste between two neighbouring aggregate surfaces in concrete $(' B$ ' = bulk, 'I' = 'ITZ). W25 = water-binder ratio $=0.25 ; F 300=$ fineness is $300 \mathrm{~mm} 2 / \mathrm{kg}$; $p=$ porosity. 
practice. Muller et al. (2013) recently showed by ${ }^{1} \mathrm{H}$ NMR relaxation analysis that for a white cement paste with a water-cement-ratio of 0.4 , the total capillary porosity was $9.2 \%$ by volume after 28 days of hydration; only $1.4 \%$ was filled by unconsumed water and the rest were 'empty' chemical shrinkage voids. They further found it difficult (if not impossible) to subsequently refill these voids (Zalzale et al., 2013).

To model permeability at various degrees of water saturation, an "emptying" algorithm was implemented. In this algorithm, water was removed progressively by evaporation from fully saturated microstructures, starting from the largest pores according to the KelvinLaplace law, following Zalzale et al. (2013). The positions and diameters of pores were determined using DRaMuTS and SVM. Next, all pores were sorted by their sizes and stored for further analysis. In this way, it is easy to find the largest pores. Once their positions were determined, some virtual spheres with the same sizes as the corresponding pores could be placed to block the original transport path for water, representing the partially saturated state, as illustrated in Fig. 12.

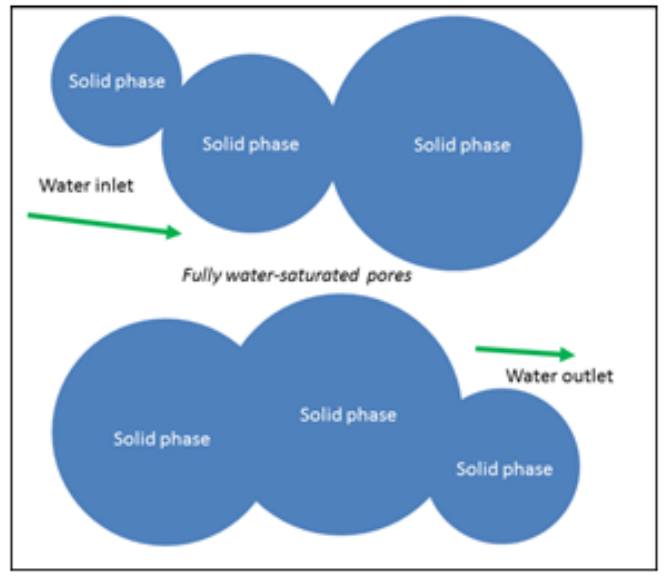

(a)

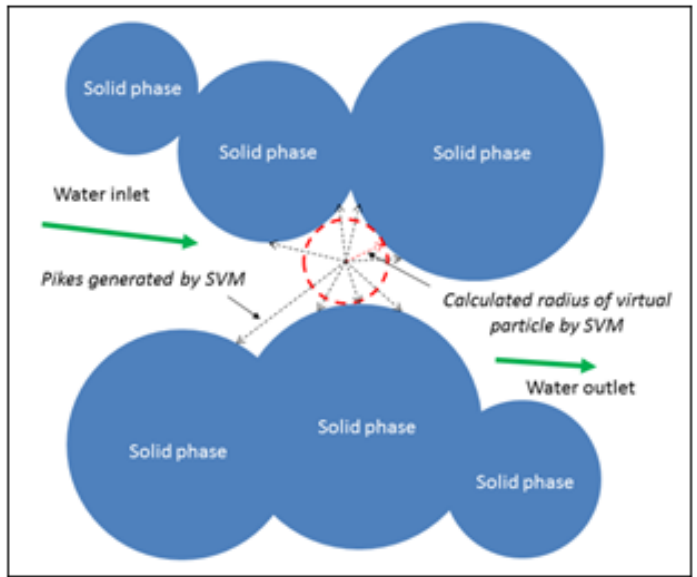

(b)

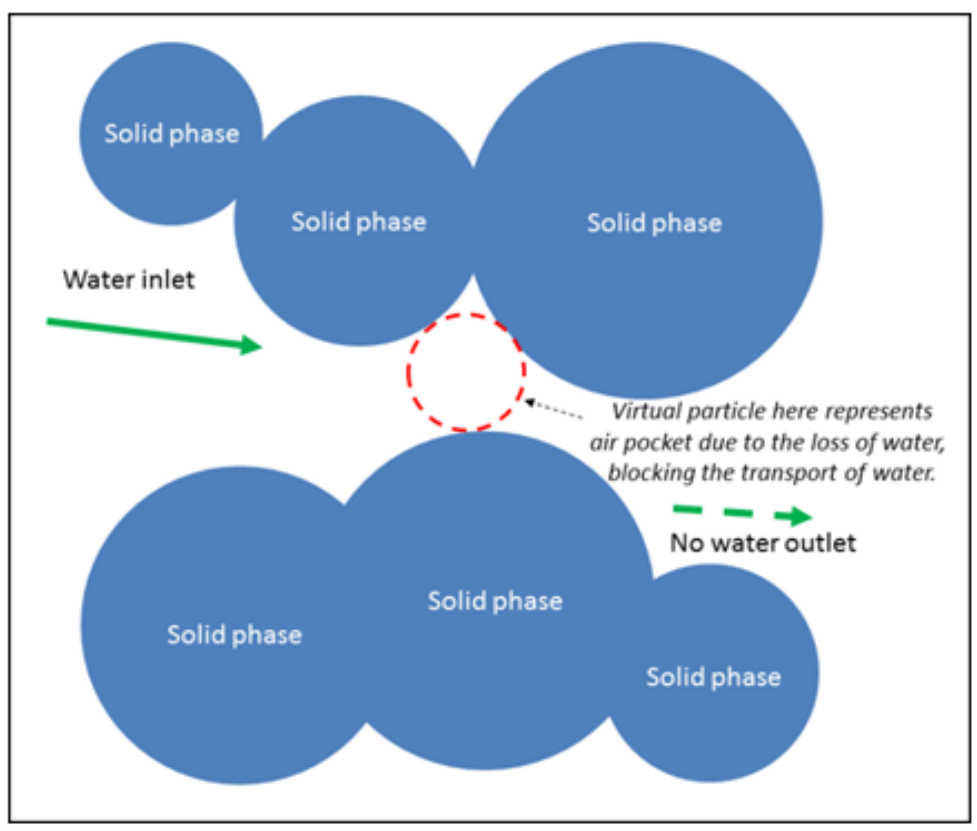

(c)

Fig. 12. Illustration of the "emptying" algorithm in 2D: (a) Fully saturated state; (b) assessment of smallest pore for positioning of a solid disc in water-filled pore. (c) Partially saturated state. The position and size of the solid disc (red circle) are obtained by DRaMuTS. 
So, the emptied pores become impermeable to water, along with the solid phase. The remaining open space is assumed still filled by water. Next, the water-filled pores of the pore system were investigated by DRaMuTS. The degree of water saturation is defined as the volume of the water-filled pores divided by the total pore volume. To avoid systematic bias due to the emptying algorithm, multiple repeats (8 samples) of the algorithm were run in different parent structures, all with the same overall capillary porosity $(9.5 \%)$. This process can ensure reliable inputs at any given saturated structure for later permeability analyses.

In the case of air penetration, pores filled by water become impermeable to gas, since gas transport can only take place in the percolated air-filled pores. The penetrative phenomenon of air in a water environment is usually too small to be taken into account. The capillary pressure in small pores hamper the water evaporation, so these pores are always easy to be filled by water, but the water will be hard to remove. Hence, these small pores become impermeable to air while the large ones open the transport path for gas because of water evaporation. So, the emptying algorithm starts from the smallest pores in the gas permeability modelling case. Since air permeability is outside the scope of this paper, the interested reader is referred to our coming publication.

\section{RESULTS}

\section{PERMEABILITY AND WATER SATURATION}

Transport properties of cementitious materials, mainly permeability, play a key role in assessing and predicting the durability of such materials.

However, people do not currently know the mechanism which controls the evolution of the permeability of cement-based materials, as the measured permeability varies widely and strongly depends on the penetrating fluid. Several hypotheses have been proposed to explain this phenomenon, such as delayed cement hydration (Dhir et al., 1989; Loosveldt et al., 2002), micro-cracks induced by sample drying prior to gas permeability measurements (Wong et al., 2009), gas slippage on the surface of the pores (Bamforth, 1987), dissolution and migration of fine elements (Banthia and Mindess, 1989; Dhir et al., 1989; Hearn et al., 1994; Loosveldt et al., 2002). But, none of these is sufficient to explain fully the wide variation of results. Coussy et al. (1998) have shown that a change in physical-mechanical properties of cementi- tious materials strongly depends on their water saturation degree (Dhir et al., 1989; Abbas et al., 1999; Wong et al., 2007; Baroghel-Bouny et al., 2011). This indicates that the variation of measured permeability may indeed be attributed to the degree of water saturation.

Actually, Abbas et al. (1999), Wong et al. (2007) and Baroghel-Bouny et al. (2011) have shown a strong dependency of the intrinsic gas permeability on the degree of water saturation for concretes, grouts and mortars. As for the water permeability, only very few experimental results have been published so far; the main reason is that it is quite difficult to establish and maintain the total saturation during the tests. Very recently, Kameche et al. (2014) found that both liquid water permeability and gas permeability of ordinary concrete are a function of the degree of water saturation.

The transport of water through a pore system that contains various amounts of stagnant water is therefore investigated by our methodology. The two prime parameters that influence permeability in partly saturated conditions are PoSD and pore connectivity. These parameters will be discussed separately. In the last part of this section, our simulation results in terms of permeability are validated by experimental data.

\section{PORE SIZE DISTRIBUTION}

PoSD and connected pore fraction are intimately connected to the structural changes in cementitious materials underlying a phenomenon as water permeability. Herein, these two parameters are investigated by DRaMuTS and SVM to link the variations of water permeability of cement paste at five different capillary water saturations $(100 \%, 73.67 \%, 53.19 \%$, $43.99 \%, 26.23 \%$ ) to its internal structural changes. In Fig. 13, it can be observed that the pore size distribution curve of cement paste shifts to the left side as the capillary water saturation decreases. Remember that water was progressively removed from the largest pores according to the Kelvin-Laplace equation to reach lower saturation degrees. As a consequence, the proportion of small pores increases with decreasing saturation degree yielding the left shift phenomenon. Fig. 14 reveals an almost linear decline in the median pore size with diminishing degree of water saturation in the pores. So, the PoSD is very sensitive to the degree of water saturation in the pores.

\section{PORE CONNECTIVITY}

The other important parameter, pore connectivity in mature cement paste, was studied as well. DRaMuTS is based on a random points distribution system, so the confidence of results relies strongly on the number 
of points. A large number of points usually leads to more accurate results, but yields excessive computational efforts and time. Therefore, a sensitivity analysis of connected pore fraction at various degrees of water saturation was carried out. The results are plotted in Fig. 15.

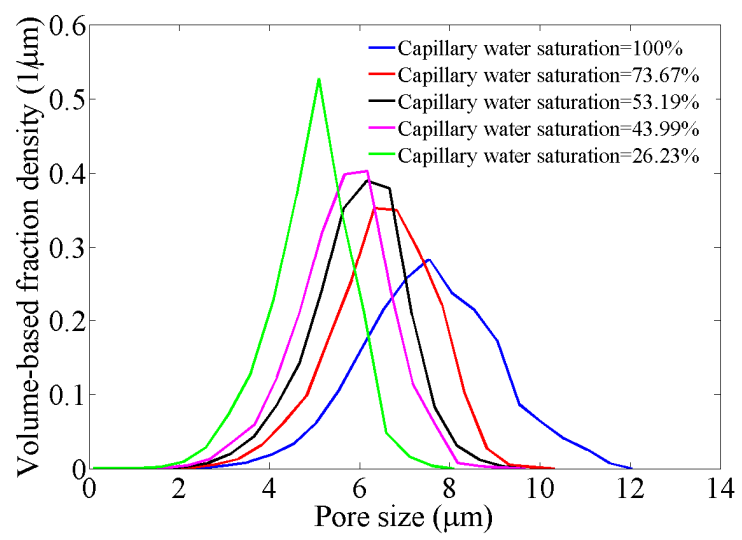

Fig. 13. Pore size distribution of samples at various degrees of water saturation.

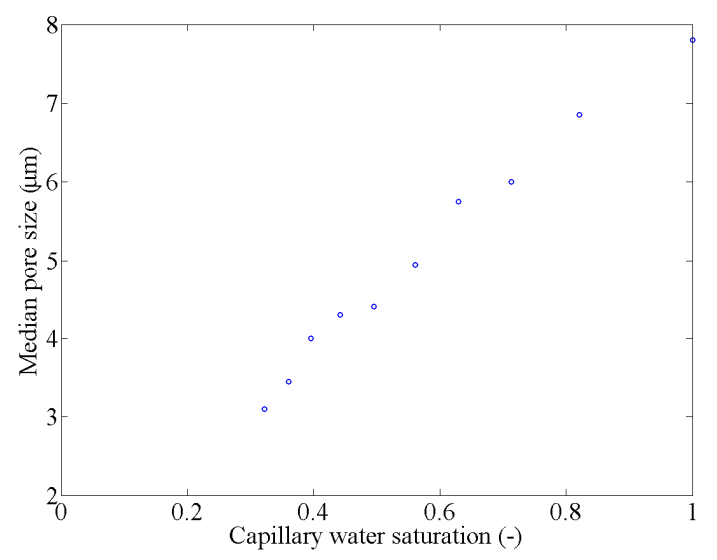

Fig. 14. Almost linear reduction in median pore size with declining degree of water saturation in the capillary pore network structure.

Obviously, connected pore fraction increases sharply as the number of points goes up, where-upon the curves bend to reach a plateau level. In the present research, $10^{5}$ points are used in all cases to minimize computation time in combination with a high accuracy level. Connected pore fraction is plotted as a function of capillary water saturation in Fig. 16. With decreasing capillary water saturation, the fully water-filled pores will gradually become blocked by air bubbles, leading to a reduced connected pore fraction of the microstructures. This rate of decline increases at further reduction of the degree of saturation, as a direct reflection of the increased spacing of the curves in Fig. 15.

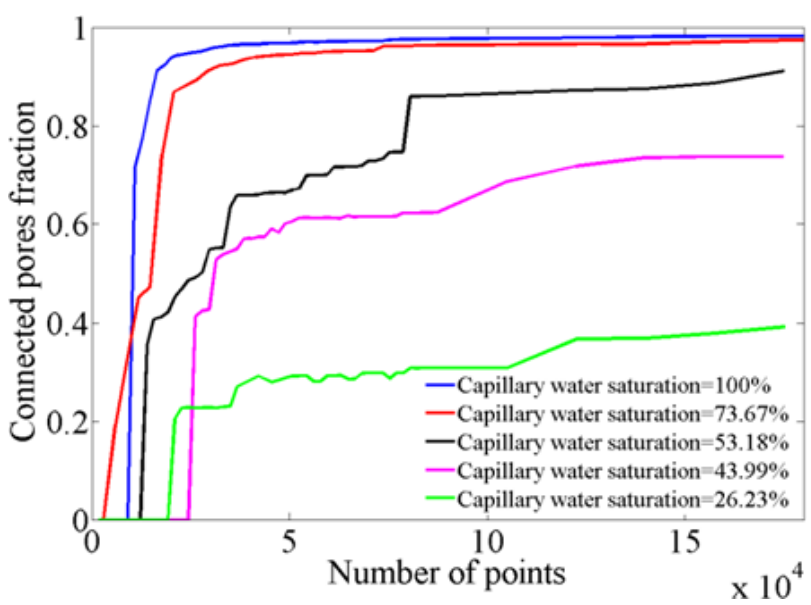

Fig. 15. Sensitivity analysis of connected pore fraction at various degrees of water saturation.

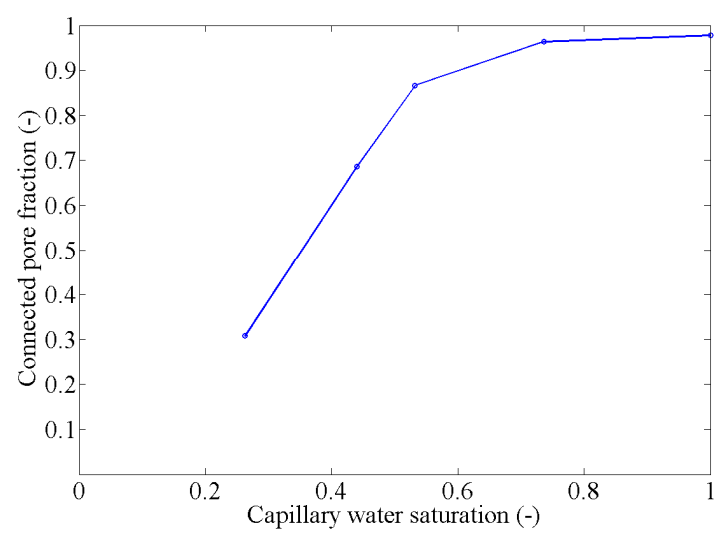

Fig. 16. Connected pore fraction as a function of capillary water saturation.

\section{VALIDATION}

To compare with the available experimental data obtained on concrete, relative permeability is plotted in Fig. 17. This is defined as the ratio of intrinsic permeability at a certain degree of saturation to intrinsic permeability measured in the fully saturated state. In the case of water permeability, a sample fully filled by water implies that the water saturation equals 1 . Obviously, an agreement between experimental data and simulation results obtained by the presented methodology is very satisfactory, validating the presented methodological approach.

It should be noted again that the extreme values of water saturation were most probably not realized in the reported experiments shifting the associated curves even more closer to the simulation results. Of course, Fig. 17 reveals the joint effects of the two discussed parameters, PoSD and connectivity. 


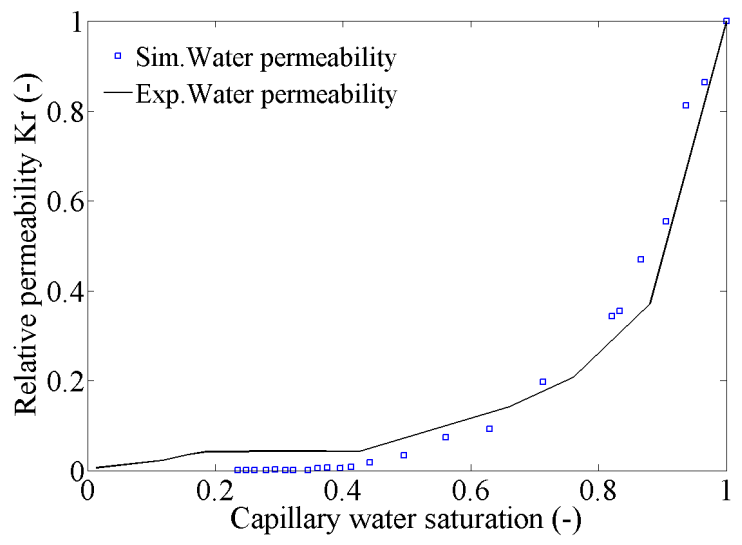

Fig. 17. Effect of stagnant water content in pore system in virtual hydrated cement paste on relative permeability $K_{r}$ for water. Validation is by experimental data for hardened concrete with various amounts of stagnant water in the pore system taken from Kameche et al. (2014).

\section{SUMMARY}

Durability is still a major problem in concrete technology. Because processes endangering concrete durability are essentially progressing slowly, research into such matters is time consuming and only reasonably possible by speeding up the processes. However, this introduces uncertainties, inevitably limiting their applicability. So, its relative expensive and timeconsuming character set a limit to realcrete research. Moreover, obtained results are not always reliable either. Hence, nowadays exploiting the possibilities of studying virtual representations of the realcrete is attractive, because less time-consuming and laborious. And as a result of the fast developments in computational facilities, this can be done in an increasingly sound way.

This paper reviews a novel methodology for producing and thereupon investigating the characteristics (that is, porosimetry and water permeability) of a reliable representation of a cementitious composite. This is due to particle packing generated by dynamic force-based DEM, contrary to random sequential addition (RSA) systems that are quite popular in concrete technology. Literature demonstrates the superiority of DEM over RSA when structure-sensitive properties are at issue (Williams and Philipse, 2003; Chen et al., 2006). Even porosity, generally assumed structureinsensitive, has been demonstrated somewhat depending on the way particles are dispersed (Hu, 2004). Pore size distribution and pore depercolation are definitely of structure-sensitive nature (Chen et al., 2006). Hence, estimates relying on such parameters (e.g., permeability) will certainly be biased when based on RSA particle packing.

None of the four "building blocks" that integrated constitute the presented porosimetry methodology are completely new. This significantly reduces the risks for failure at the very end of the line. Frequently use is made of experiences and methods outside the field of concrete technology. Robotics literature (LaValle and Kuffner, 2001) rendered possible to develop DRaMuTS, while literature in spatial statistics and life sciences (Gundersen et al., 1988) provided us with the SVM approach to pore measuring. Tube network modelling for permeability estimation is quite established as well (Vogel and Roth, 2001; $\mathrm{Hu}, 2004$; Chiu et al., 2013), so that also in this case we could make use of earlier experiences (outside the field of concrete technology).

Earlier objections toward outcomes of the porosimetry approach (pores would be too large when produced by vector-based methods) are primarily resulting from an incorrect interpretation of experiments, whereby the method is at stake (MIP versus quantitative image analysis), or the interpretation is incorrect (experiments using specimens which are not fully saturated even when pursued). The agreement between simulation results and experiments on partly saturated concrete specimens is very satisfactory.

Nevertheless, the vector-based approach produces smooth-surfaced pores. This is indeed a deficiency of this method. We have demonstrated however that the outer layer of hydration products could be simulated by means of particle packing on nano-level. In doing so, a fibrous structure of CSH could be simulated in the outer hydration layer in agreement with experimental observations by Richardson (2004). This would yield a rough surface, additionally slightly diminishing pore size. Further, pore continuity would be reduced, so that a lower conductance is obtained. Both reductions in section conductance as well as pore continuity would result in lower permeability and could be used to eliminate the shortcomings of the vector approach. However, at this stage of the developments it is believed that this may only be of marginal importance until further and improved experimental approaches will prove the contrary.

The various building blocks jointly forming the presented methodology have been individually validated and laid down in a series of publications. Moreover, such blocks were obtained by upgrading of available methodology used in other branches of science, so making use of positive experiences or even of a mathematical foundation. 
The methodology is presently exploited for systematically investigating effects of technological and environmental parameters on durability capacity (when based, of course, on transport through the capillary pores). The relevant results of such technological studies will be published elsewhere.

\section{REFERENCES}

Abbas A, Carcasses M, Ollivier JP (1999). Gas permeability of concrete in relation to its degree of saturation. Mater Struct 32:3-8.

Abell AB, Willis KL, Lange DA (1999). Mercury intrusion porosimetry and image analysis of cement-based materials. J Colloid Interf Sci 211:39-44.

Bamforth PB (1987). The relationship between permeability coefficients for concrete obtained using liquid and gas. Mag Concr Res 39:3-11.

Banthia N, Mindess S (1989). Water permeability of cement paste. Cem Concr Res 19:727-36.

Baroghel-Bouny V, Thiery M, Wang X (2011). Modelling of isothermal coupled moisture-ion transport in cementitious materials. Cem Concr Res 41:828-41.

Chen H, Stroeven P, Ye G, Stroeven M (2006). Influence of boundary conditions on pore percolation in model cement paste. Key Engr Mat 302-303:486-92.

Chiu SN, Stoyan D, Kendall WS, Mecke J (2013). Stochastic geometry and its applications, 3rd Ed. Chichester: Wiley.

Coussy O, Eymard R, Lassabatere T (1998). Constitutive unsaturated modeling of drying deformable materials. J Eng Mech 124:658-67.

Diamond S (2000). Mercury porosimetry: an inappropriate method for the measurement of pore size distribution in cement-based materials. Cem Concr Res 30:1517-25.

Dhir RK, Hewlett PC, Chan YN (1989). Near surface characteristics of concrete: intrinsic permeability. Mag Concr Res 41:87-97.

Garboczi EJ, Bentz, DP (2001). The effect of statistical fluctuation, finite size error, and digital resolution on the phase percolation and transport properties of the NIST cement hydra-tion model. Cem Concr Res 31: 1501-14.

Garboczi EJ, Bullard JW (2004). Shape analysis of a reference cement. Cem Concr Res 34:1933-7.

Gundersen HJG, Bendtsen TF, Korbo L, Marcussen N, Møller A, Nielsen K, et al. (1988). Some new, simple and efficient stereological methods and their use in pathological research and diagnosis. APMIS 96:379-94.

$\mathrm{He} \mathrm{H}$ (2010). Computational modelling of particle packing in concrete. PhD Thesis, Delft University of Technology. Delft.

He H, Stroeven P, Stroeven M and Sluys LJ. (2011). Influence of particle packing on fracture properties of concrete. Comp Concr 8:677-92.
Hearn N, Detwiler RJ, Sframeli C (1994). Water permeability and microstructure of three old concretes. Cem Concr Res 24:633-40.

Hu J, Stroeven P (2003). Application of image analysis to assessing critical pore size for permeability prediction on cement paste. Image Anal Stereol 22: 7-103.

Hu J (2004). Porosity of concrete: Morphological study of model concrete. PhD Thesis, Delft University of Technology. Delft.

Hu J, Stroeven P (2006). Shape characterization of concrete aggregate. Image Anal Stereol 25:43-53.

Kameche ZA, Ghomari F, Choinska M, Khelidj A (2014). Assessment of liquid water and gas permeabilities of partially saturated ordinary concrete. Construct Build Mater 65:551-65.

Lange DA, Jennings HM, Shah SP (1994). Image analysis techniques for characterization of pore structure of cement-based materials. Cem Concr Res 24:841-53.

LaValle SM and Kuffner JJ (2001). Rapidly-exploring random trees:progress and prospects. In:Donald BR, Lynch KM and Rus D, eds. Algorithmic and computational robotics: New directions. Wellesley (Ma).

Le LBN (2015). Micro-level porosimetry of virtual cementitious materials - Structural impact on mechanical and durability evolution. PhD Thesis, Delft University of Technology. Delft.

Le LBN and Stroeven P (2014). Packing issue in cement blending for sustainability developments - Approach by discrete element method. Int J Res Eng Technol 3:89-96.

Le LBN, Stroeven M, Sluys LJ, Stroeven P (2013). A novel numerical multi-component model for simulating hydration of cement. Comp Mater Sci 78:12-21.

Le LBN, Stroeven P (2012). Porosity of green concrete based on a gap-graded blend. In:Brandt AM, Olek MA and Leung CKY, eds. Proceedings of the International Symposium on Brittle Matrix Composites 10, 2012 October 15-17; Warsaw, Poland, 315-24.

Li K, Le LBN, Stroeven P, Stroeven M (2014). Strategy for predicting transport-based dura-bility properties of concrete based on DEM approach. In: Bjegovic D, Beushausen H, Serdar M, eds. Proceedings of the RILEM International Workshop on Performance-based Specification and Control of Concrete Durability, 2014 June 11-13; Zagreb, Croatia, 443-50.

Loosveldt H, Lafhaj Z, Skoczylas F (2002). Experimental study of gas and liquid permeability of a mortar. Cem Concr Res 32:1357-63.

Mason G and Morrow NR (1991). Capillary behaviour of a perfectly wetting liquid in iregular triangular tubes. J Colloid Interf Sci 141:262-74.

Muller ACA, Scrivener KL, Gajewicz AM, McDonnald PJ (2013). Densification of C-S-H measured by 1H NMR relaxometry. J Phys Chem C 117:403-12. 
Patzek TW, Silin DB (2001). Shape factor and hydraulic conductance in noncircular capillaries: One-phase creeping flow. J Colloid Interf Sci 236:295-304.

Pignat C, Navi P, Scrivener K (2005). Simulation of cement paste microstructure, hydration, pore space characterization and permeability determination. Mat Struct 38: 450-66.

Richardson IG (2004). Tobermorite/jennite- and tobermorite/ calcium hydroxide-based models for the structure of C-S-H:Applicability to hardened pastes of tricalcium silicate, $\beta$-dicalcium silicate, Portland cement, and blends of Portland cement with blast-furnace slag, metakaolin, or silica fume. Cem Concr Res 34:1733-77.

Scrivener KL (1989). The use of backscattered electron microscopy and image analysis to study the porosity of cement paste. In: Roberts LR, Skalny JP, eds. Proceedings of material research society symposium 137 , 1989; Warrendale, PA, 129-40.

Stroeven P, Hu J, Guo Z (2009). Shape assessment of particles in concrete technology: 2D image analysis and 3D stereological extrapolation. Cem Concr Compos 31: 84-91.

Stroeven P, Hu J, Koleva DA (2010). Concrete porosimetry: Aspects of feasibility, reliability and economy. Cem Concr Compos 32:291-99.

Stroeven P, He H, Stroeven M (2011). Discrete element approach to assessment of granular properties in concrete. J Zhejiang Univ - Sci A 12:335-44.

Stroeven P, Le LBN, Sluys LJ, He H (2012a). Porosimetry by double random multiple tree structuring. Image Anal Stereol 31:55-63.

Stroeven P, Le LBN, Sluys LJ, He H (2012b). Porosimetry by random node structuring in virtual concrete. Image Anal Stereol 31:79-87.

Stroeven P, Le LBN (2013). Evaluation by discrete element method (DEM) of gap-graded packing potentialities for green concrete design. In: Soustos M, Goodier C,
Nguyen VT, eds. The International Conference on Sustainable Built Environment for Now and the Future, 2013 March 26-27; Hanoi, Vietnam, 347-54.

Vogel HJ and Roth K (2001). Quantitative morphology and network representation of soil pore structure. Adv Water Resour 24:233-44.

Wang Y, Diamond S (1995). An approach to quantitative image analysis for cement pastes. In: Diamond $\mathrm{S}$, Mindess S, Glasser FP, Roberts LW, Skalny JP, Wakeley LD, eds. Microstructure of cement based systems/ bonding and interfaces in cementitious materials, Vol. 370 Material Research Society, Pittsburgh, 23-32.

Willis KL, Abell AB and Lange DA. (1998). Image-based characterization of cement pore structure using wood's metal intrusion. Cem Concr Res 28:1675-1705.

Williams SR and Philipse AP (2003). Random packings of spheres and spherocylinders simu-lated by mechanical contraction. Phys Rev E 67:1-9.

Wong H, Buenfeld N, Hill J, Harris A (2007). Mass transport properties of mature wasteform grouts. Adv Cem Res 19:35-46.

Wong HS, Zobel M, Buenfeld NR, Zimmerman RW (2009). Infulence of the interfacial transition zone and microcracking on the diffusivity, permeability and sorptivity of cement-based materials after drying. Mag Concr Res 61:571-89.

Ye G (2003). Experimental study and numerical simulation of the development of the microstructure and permeability of cementitious materials. PhD Thesis, Delft University of Technology. Delft.

Zalzale M, McDonnald PJ, Scrivener KL (2013). A 3D lattice Boltzmann effective media study:understanding the role of C-S-H and water saturation on the permeability of cement paste. Modelling Simul Mater Sci Eng 21:085016. 